\title{
ABSOLUTELY STRUCTURALLY STABLE DIFFEOMORPHISMS ${ }^{1}$
}

\author{
JOHN FRANKS
}

\begin{abstract}
This paper gives a proof that if a diffeomorphism is structurally stable in a strong sense then it satisfies Axiom A of S. Smale. This provides a weakened converse of a theorem of J. Robbin on structural stability.
\end{abstract}

In this note we prove some results on structurally stable diffeomorphisms analogous to recent results on $\Omega$-stability in [2] and [4]. Recall that a diffeomorphism $f: M \rightarrow M$ of a compact manifold is said to be structurally stable if there is a neighborhood $N$ of $f$ in $\operatorname{Diff}^{1}(M)$ with the property that to each $g \in N$ there corresponds a homeomorphism $h$ of $M$ such that $g \circ h=$ $h \circ f$. In [5], J. Robbin proves a conjecture of Smale which provides sufficient conditions for $f$ to be structurally stable when $f$ is $C^{2}$. In this paper we show that if the definition of structurally stable is strengthened these conditions are necessary as well as sufficient.

Definition. A diffeomorphism $f: M \rightarrow M$ is absolutely structurally stable if there is a neighborhood $N$ of $f$ in $\operatorname{Diff}^{1}(M)$ and a function $\phi: N \rightarrow$ $C^{0}(M, M)$ such that:

(1) $\phi(g)$ is a homeomorphism for each $g \in N$, and $\phi(f)=\mathrm{id}: M \rightarrow M$.

(2) $g \circ \phi(g)=\phi(g) \circ f$.

(3) There is a constant $K>0$ such that

$$
\sup _{x \in M I} d(\phi(g)(x), x) \leqq K \sup _{x \in M I} d(f(x), g(x)),
$$

where $d$ is a metric on $M$.

THEOREM 1. If $f: M \rightarrow M$ is $C^{2}$ and $M$ is compact then $f$ is absolutely structurally stable if and only if $f$ satisfies Axiom A and the strong transversality property.

We remark that the requirement that $f$ be $C^{2}$ is necessary for only one direction of the implication. Even if $f$ is only $C^{1}$ it is still true that absolute structural stability implies Axiom A and the strong transversality property.

Received by the editors March 16, 1972.

AMS (MOS) subject classifications (1970). Primary 58F10; Secondary 58F15.

${ }^{1}$ Work supported by National Science Foundation Grant GP-19815. 
In [5], Robbin proves that if $f$ is $C^{2}$ and satisfies Axiom A and the strong transversality property then a function $\phi$ satisfying (1) and (2) of the definition above exists and is continuous. However, a stronger statement holds.

THEOREM 2. If $f$ is $C^{2}$ and is absolutely structurally stable then $\phi$ and $N$ can be chosen so that $\phi: N \rightarrow C^{0}(M, M)$ is a $C^{1}$ map.

Proof of Theorem 1. We first show that Axiom A and strong transversality imply absolute structural stability. The continuous maps from $M$ to itself, $C^{0}(M, M)$, have the structure of a $C^{\infty}$ Banach manifold. We will identify the tangent space to $C^{0}(M, M)$ at id with $\Gamma^{0}$, the space of continuous sections of the tangent bundle $T M$ (see [1] or [3] for this). $\Gamma^{0}$ is a Banach space with the sup norm.

Let exp: $T M \rightarrow M$ be the exponential map arising from a $C^{\infty}$ Riemannian metric on $M$; then there is an open neighborhood $U$ of 0 in $\Gamma^{0}$ such that the map $\Psi: U \rightarrow C^{0}(M, M)$ given by $\Psi(\gamma)=\exp \circ \gamma$ defines a $C^{\infty}$ chart on $C^{0}(M, M)$. Note that if $h=\Psi(\gamma)$ then $\sup _{x \in, M} d(h(x), x)=\|\gamma\|$, where \|\| is the sup norm on $\Gamma^{0}$ and also $D \Psi(0): \Gamma^{0} \rightarrow \Gamma^{0}$ is the identity.

We define the adjoint map $f^{\#}: \Gamma^{0} \rightarrow \Gamma^{0}$ by $f^{\#}(\gamma)=d f^{-1} \circ \gamma \circ f$ (this coincides with the definition of $f^{\#}$ in [5] but is the inverse of the $f^{\#}$ defined in [2]).

In [5], Robbin shows that if $f$ is $C^{2}$ and satisfies Axiom $A$ and the strong transversality condition then there is a neighborhood $\dot{N}$ of $f$ in $\operatorname{Diff}^{1}(M)$, and a linear map $J: \Gamma^{0} \rightarrow \Gamma^{0}$ satisfying

(1) $\left(I-f^{\#}\right) \circ J=I$, the identity on $\Gamma^{0}$, and

(2) for each $g \in N$ there is a unique point, $\hat{\phi}(g)$, in $U$ กimage $J$ which has the property that if $h=\Psi(\hat{\phi}(g))=\exp \circ \hat{\phi}(g)$ then $h$ is a homeomorphism and $g^{-1} \circ h \circ f=h$. Also $\hat{\phi}: N \rightarrow \Gamma^{0}$ is continuous.

If we define $\phi(g)$ to be $\Psi(\hat{\phi}(g))=\exp \circ \hat{\phi}(g)$ then $\phi$ satisfies (1) and (2) of the definition of absolute structural stability so we need only show that (3) holds.

Since $\left(I-f^{\#}\right) \circ J=I$, if $R$ is the image of $J, R$ is a closed subspace of $\Gamma^{0}$ and is a complement to the kernel of $\left(I-f^{\#}\right)$. Also $\left(I-f^{\#}\right): R \rightarrow \Gamma^{0}$ is an isomorphism. Define $F: C^{0}(M, M) \rightarrow C^{0}(M, M)$ by $F(h)=f^{-1} \circ h \circ f$ and define $\hat{F}: U^{\prime} \rightarrow \Gamma^{0}$ by $\hat{F}(\gamma)=\Psi^{-1} \circ F \circ \Psi$ where $U^{\prime}$ is a suitably chosen neighborhood of 0 in $U$. Let $W=R \cap U$, then $(I-\hat{F}): W \rightarrow \Gamma^{0}$ is a $C^{1}$ map and its derivative at 0 is $\left(I-f^{\#}\right): R \rightarrow \Gamma^{0}$ (for this see [3] or [1, p. 780]). Since $\left(I-f^{\#}\right): R \rightarrow \Gamma^{0}$ is an isomorphism by the inverse function theorem there is a neighborhood $W^{\prime}$ of 0 in $W$ on which $I-\hat{F}$ is a diffeomorphism. Hence there is a constant $q>0$ such that $\|(I-\hat{F})(\gamma)\| \geqq q\|\gamma\|$ for all $\gamma \in W^{\prime}$, so $\|\gamma\| \leqq q^{-1}\|\gamma-\hat{F}(\gamma)\|$. Since $\exp$ is $C^{1}$ there is a constant $K_{1}>0$ such that $\|\gamma-\hat{F}(\gamma)\| \leqq K_{1} \sup _{x \in M} d(\exp (\gamma(x)), \exp (\hat{F}(\gamma)(x)))$ if $\|\gamma\|$ is sufficiently 
small. Hence if $N$ is a sufficiently small neighborhood of $f$ in $\operatorname{Diff}^{1}(M), g \in N$, and we let $h=\phi(g)$ and $\gamma=\Psi^{-1}(\phi(g))$ we have

$$
\begin{aligned}
\sup _{x \in M} d(h(x), x) & =\|\gamma\| \leqq q^{-1}\|\gamma-\hat{F}(\gamma)\| \\
& \leqq K_{1} q^{-1} \sup _{x \in M I} d(F(h)(x), h(x)) \\
& =K_{1} q^{-1} \sup _{x \in M I} d\left(f^{-1} \circ h \circ f(x), h(x)\right) \\
& =K_{1} q^{-1} \sup _{x \in M I} d\left(f^{-1} \circ h \circ f(x), g^{-1} \circ h \circ f(x)\right) \\
& =K_{1} q^{-1} \sup _{x \in M I} d\left(f^{-1}(x), g^{-1}(x)\right) .
\end{aligned}
$$

But $\sup _{x \in M} d\left(f^{-1}(x), g^{-1}(x)\right)=\sup _{x \in M I} d\left(f^{-1} \circ g(x), x\right)$ and since $f^{-1}$ is $C^{1}$ there is a constant $K_{2}$ such that $d(x, y) \leqq K_{2} d(f(x), f(y))$ for all $x, y \in M$. Thus if $K=K_{1} K_{2} q^{-1}$,

$$
\sup _{x \in M} d(h(x), x) \leqq K \sup _{x \in M} d(g(x), f(x))
$$

as was to be shown.

To complete the proof of Theorem 1 we note that if $f$ is absolutely structurally stable it is a fortiori absolutely $\Omega$-stable so by the results of [2] and [4] it satisfies Axiom A and by a theorem asserted in [6] which is not difficult to prove, a structurally stable diffeomorphism which satisfies Axiom A also satisfies the strong transversality condition. Q.E.D.

Proof OF THEOREM 2. We use the same terminology as in the proof of Theorem 1. By Theorem 1, $f$ and hence also $f^{-1}$ will satisfy Axiom A and the strong transversality property so we can again cite the results of Robbin [5], this time applied to $f^{-1}$. Namely, there is a neighborhood $N^{\prime}$ of $f^{-1}$ in $\operatorname{Diff}^{1}(M)$ and a linear map $J: \Gamma^{0} \rightarrow \Gamma^{0}$ such that $\left(I-f^{\#-1}\right) \circ J=I$ and if $g \in N^{\prime}$ there is a unique $\gamma \in R$, the image of $J$, such that $g \circ h \circ f^{-1}=h$ if $h=\exp \circ \gamma$.

Now let $K$ be the kernel of $I-f^{\#-1}$ and recall that $\Gamma^{0}=K \oplus R$. If $N_{0}$ is a sufficiently small neighborhood of $f$ in $\operatorname{Diff}^{1}(M)$ then the map $H: N_{0} \times K \times$ $R \rightarrow \Gamma^{0}$, given by $H\left(g, \gamma_{1}, \gamma_{2}\right)=\gamma_{2}-\Psi^{-1}\left(g \circ \Psi\left(\gamma_{1}, \gamma_{2}\right) \circ f^{-1}\right)$ is well defined when $\left\|\gamma_{1}\right\|$ and $\left\|\gamma_{2}\right\|$ are small enough. $\Psi$ is a $C^{\infty}$ chart and the map $N_{0} \times$ $C^{0}(M, M) \rightarrow C^{0}(M, M)$ which sends $(g, h)$ to $g \circ h \circ f^{-1}$ is $C^{1}$ (see [1]), so $H$ is a $C^{1}$ map. It also follows from the results of [1] that the partial $D_{3} H(f, 0,0): R \rightarrow \Gamma^{0}$ is just $I-f^{\#-1}$ which is an isomorphism since $J$ is its inverse. Hence by the implicit function theorem there is a neighborhood $V$ of $(f, 0)$ in $N_{0} \times K$ and a unique $C^{1}$ map $\theta: V \rightarrow R$ such that $H\left(g, \gamma_{1}, \theta\left(g, \gamma_{1}\right)\right)=$ 0 for all $\left(g, \gamma_{1}\right) \in N_{0} \times K$. Let $N=\{g \mid(g, 0) \in V\}$ and let $\hat{\phi}: N \rightarrow R$ be given 
by $\hat{\phi}(g)=\theta(g, 0)$, then $\hat{\phi}$ is $C^{1}$ and $H(g, 0, \hat{\phi}(g))=0$ so $\hat{\phi}(g)=$ $\Psi^{-1}\left(g \circ \Psi(\hat{\phi}(g)) \circ f^{-1}\right)$. But if $\gamma$ is section corresponding to $g^{-1}$ which is guaranteed by the result of Robbin cited above then $\gamma \in R$ and $\gamma=$ $\Psi^{-1}\left(g \circ \Psi(\gamma) \circ f^{-1}\right)$ so by uniqueness of $\hat{\phi}(g), \gamma=\hat{\phi}(g)$. Thus if we define $\phi=\Psi \circ \hat{\phi}$, then $\phi$ is a $C^{1}$ map but also if $g \in N, \phi(g)=\exp \circ \gamma=h$, a homeomorphism satisfying $g \circ h \circ f^{-1}=h$. Q.E.D.

\section{BIBLIOGRAPHY}

1. J. Eells, A setting for global analysis, Bull. Amer. Math. Soc. 72 (1966), 751-807. MR 34 \#3590.

2. J. Franks, Differentiably $\Omega$-stable diffeomorphisms, Topology 11 (1972), 107-113.

3. - Manifolds of $C^{r}$ mappings (to appear).

4. J. Guckenheimer, Absolutely $\Omega$-stable diffeomorphisms, Topology 11 (1972), 195-197.

5. J. Robbin, A structural stability theorem, Ann. of Math. (2) 11 (1971), 447-493.

6. S. Smale, Notes on differentiable dynamical systems, Proc. Sympos. Pure Math., vol. 14, Amer. Math. Soc., Providence, R.I., 1970, pp. 277-287. MR 42 \#1152.

NORTHWESTERN UNIVERSITY, Evanston, IlLINOIS 60201 